\title{
A TIME-INDEPENDENT FINITE DIFFERENCE ANALYSIS OF VISCOUS FLOW ACROSS A TRANSLATING CIRCULAR CYLINDER
}

\author{
Chih-Chun Chu \\ Yi-Hsiang Yu \\ Bang-Fuh Chen \\ chenbf@mail.nsysu.edu.tw \\ Tin-Kan Hung \\ Department of Bioengineering, University of Pittsburgh, Pittsburgh, PA, USA.
}

Department of Marine Environment and Engineering, National Sun Yat-sen University, Kaohsiung, Taiwan, R.O.C

Department of Marine Environment and Engineering, National Sun Yat-sen University, Kaohsiung, Taiwan, R.O.C

Department of Marine Environment and Engineering, National Sun Yat-sen University, Kaohsiung, Taiwan, R.O.C,

Follow this and additional works at: https://jmstt.ntou.edu.tw/journal

Part of the Engineering Commons

\section{Recommended Citation}

Chu, Chih-Chun; Yu, Yi-Hsiang; Chen, Bang-Fuh; and Hung, Tin-Kan (2010) "A TIME-INDEPENDENT FINITE DIFFERENCE ANALYSIS OF VISCOUS FLOW ACROSS A TRANSLATING CIRCULAR CYLINDER," Journal of Marine Science and Technology: Vol. 18: Iss. 4, Article 18.

DOI: $10.51400 / 2709-6998.1928$

Available at: https://jmstt.ntou.edu.tw/journal/vol18/iss4/18

This Research Article is brought to you for free and open access by Journal of Marine Science and Technology. It has been accepted for inclusion in Journal of Marine Science and Technology by an authorized editor of Journal of Marine Science and Technology. 


\section{A TIME-INDEPENDENT FINITE DIFFERENCE ANALYSIS OF VISCOUS FLOW}

ACROSS A TRANSLATING CIRCULAR CYLINDER

\section{Acknowledgements}

This study is partially supported by Nation Science Council of ROC under a grant NSC-90-2611-E-110-001 and NSC-96- 2211-E-110-107-MY3. 


\title{
A TIME-INDEPENDENT FINITE DIFFERENCE ANALYSIS OF VISCOUS FLOW ACROSS A TRANSLATING CIRCULAR CYLINDER
}

\author{
Chih-Chun Chu*, Yi-Hsiang Yu*, Bang-Fuh Chen*, and Tin-Kan Hung**
}

Key words: viscous flow, circular cylinder, time-independent finite difference method.

\section{ABSTRACT}

A time-independent finite difference scheme is developed and applied to analyze the viscous flow past a translating circular cylinder. In this scheme, the instantaneous moving cylinder face is mapped to a fixed boundary and the computational domain is, therefore, time independent. Large Reynolds number and high oscillating frequency of moving cylinder are used in the analysis. The finite difference approximation and algorithm were validated by the reported numerical simulation and flow visualization. The vorticity and streamline patterns developments were described and discussed. The surface vorticity distribution and position of separation point versus phases of oscillating stages were discussed. The flow behaviors of various amplitudes of exciting velocity and frequencies of moving cylinder are simulated and compared. The in-line forces on cylinder during cylinder oscillation were calculated and are in good agreement with those predicted by Morison's equation. The developed scheme can be extended to analyze the viscous flow passing an arbitrary moving cylinder.

\section{INTRODUCTION}

The viscous flow across a circular cylinder has draw significant attention during the last two decades. In the 20th century, the earliest study of flow around circular cylinder was reported by Blasius in [1], who used boundary layer theory to give a second order time series solution. Since then, many researchers spent their efforts to find higher order solutions and discussed the flow patterns behind the separation points. Among those, the most important investigations could be cred-

Paper submitted 08/25/09; revised 11/06/09; accepted 11/25/09. Author for correspondence: Bang-Fuh Chen (e-mail: chenbf@mail.nsysu.edu.tw).

*Department of Marine Environment and Engineering, National Sun Yat-sen University, Kaohsiung, Taiwan, R.O.C.

**Department of Bioengineering, University of Pittsburgh, Pittsburgh, PA, USA. ited to Collins \& Dennis [6, 7], they developed analytical theory and implemented their theory numerically to solve for vorticity equation up to third order solutions. The later numerical studies almost used these two presentations to validate their simulations. The first experimental study was made by Prandtl as early as [12]. Bouard and Coutanceau [2] made a much more detailed visualization and gave a detailed description of the streamline patterns development for various Reynolds numbers, $\left(R e=2 a v_{o} / v\right.$, in which $v_{o}=$ the velocity of the cross flow, $v$ the kinematic viscosity of fluid and $a$ the radius of the cylinder) and the mechanism of the phenomenon $\alpha$ and $\beta$. The numerical method used to solve the problem was first studied by Payne in [11] for $R e=40$ to 100 . After then, various numerical methods were developed to solve the problems for higher Reynolds number. Such as Ta Phuoc Loc [13] used fourth-order finite difference scheme for $R e=550-1$, 100, Ta Phuoc Loc and Bouard [14] for $R e=3,000$ to 9,500, Chang and Chern [3] used hybrid vortex method to give the solution for $R e$ up to $10^{6}$.

The interaction between impulsive started cross flow and moving cylinder are seldom presented in the literatures. In a study of Dütsch et al. [8], the low Reynolds number (100 to 200 ) and low KC number (5 to 10) were assumed. The corresponding oscillating period of cylinder is $5 \mathrm{sec}$. or the exciting frequency is as small as $0.2(1 / \mathrm{sec})$ only. However, the well known El Centro 1940 earthquake presented the peak ground acceleration of $0.32 \mathrm{~g}$, the maximum ground velocity of $0.35 \mathrm{~m} / \mathrm{sec}(\operatorname{Re}=35,000)$, and the exciting period of $0.25-0.6 \mathrm{sec}$. The frequency is as large as 1.6 to $4(1 / \mathrm{sec})$ that are about 8 to 20 times larger than that used in Dütsch's study. In order to simulate flow across an offshore cylinder in a real marine environment during earthquakes not only large Reynolds number but also the high oscillating frequency are needed in the analysis. Under earthquakes, the relative velocity between cross flow (current) and moving cylinder (induced by ground motion) may be very large and the flow is actually turbulent. The analysis of turbulent flow phenomenon requires proper turbulence model and is beyond the scope of the present study.

In the present study, the cylinder is oscillating with high frequency under a cross flow of $R e=3,000$. The finite difference approximation and algorithm were validated by the 
reported visualization. The $\mathrm{KC}$ number used in the present study is small and no artificial numerical disturbance (Justesen [10]) is introduced, the symmetrical flow, therefore, remains throughout the simulation. Detail streamline patterns are described. The positions of separation point versus phases are discussed. The results of various amplitudes of exciting velocity are compared. The in-line forces on cylinder during cylinder oscillation were calculated. The application and limitation of Morison's equation was discussed.

The main focus of this paper is the introduction of the application of time-independent finite difference technique on viscous flow cylinder interaction. The flow phenomenon of involved in this study is, therefore, briefly discussed in the paper. Section II gives brief description of the governing equations used in the study and section III introduces the numerical method and provides some references of adopted numerical algorithm. Section IV presents the results obtained in this study and section V summarizes the key conclusions.

\section{BASIC EQUATIONS}

In the present study, we assume the cross flow and cylinder are suddenly starts to move in an incompressible viscous fluid. The cross flow is moving with a constant speed $v_{o}$ and the cylinder is stream-wise oscillating. The motion in a twodimensional is described in terms of the two simultaneous equations satisfied by the stream function and the scalar vorticity. One is the stream function equation, written in polar coordinate $(\tilde{r}, \theta)$ system, as

$$
\tilde{\omega}=-\left(\nabla^{2} \psi\right)=-\left[\frac{1}{\tilde{r}} \frac{\partial}{\partial \tilde{r}}\left(\tilde{r} \frac{\partial \psi}{\partial \tilde{r}}\right)+\frac{1}{\tilde{r}^{2}} \frac{\partial^{2} \psi}{\partial \theta^{2}}\right]
$$

and the other is the so called vorticity transport equation written as

$$
\frac{\partial \tilde{\omega}}{\partial t}-\frac{1}{\tilde{r}}\left(\frac{\partial \psi}{\partial \theta} \frac{\partial \tilde{\omega}}{\partial \tilde{r}}-\frac{\partial \tilde{\omega}}{\partial \theta} \frac{\partial \psi}{\partial \tilde{r}}\right)=v \nabla^{2} \tilde{\omega}
$$

where $\psi$ is the stream function; and $\tilde{w}$ is the vorticity, and $v$ represents the kinematic viscosity. The definition sketch of the problem is conceptually shown in Fig. 1, where $d$ is the diameter of the cylinder, $a_{\mathrm{r}}$ is the distance between the outer boundary and the origin of the polar coordinate system. $d=2 a$ $a$ is the radius of cylinder. $\vec{r}^{\prime}$ is the radial distance measured from the instant center of moving cylinder and $\phi$ is the angle between $\vec{r}^{\prime}$ and horizontal direction and among those parameters, the following relationship can be given

$$
\tilde{r} \cos \theta=\delta_{x}+r^{\prime} \cos \phi
$$

in which $\delta_{x}$ is the corresponding horizontal displacements of the cylinder in stream-wise direction. The corresponding flow velocity in $\vec{r}^{\prime}$ and $\phi$ directions are defined as $v_{r^{\prime}}$ and $v_{\phi}$ and

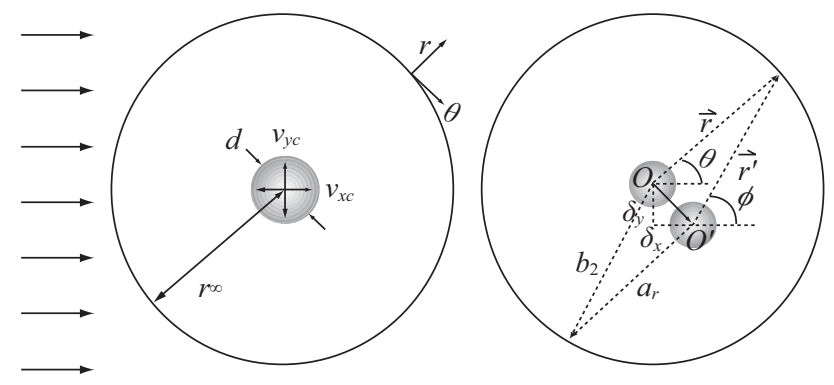

Fig. 1. The definition of sketch of the problem.

can be calculated through the definitions of velocity and stream function;

$$
v_{r^{\prime}}=\frac{1}{r^{\prime}} \frac{\partial \psi}{\partial \phi}, \quad v_{\phi}=-\frac{\partial \psi}{\partial r^{\prime}}
$$

The no-slip boundary condition assures the boundary condition of the stream function is zero when the cylinder is fixed. When the cylinder is in motion, the corresponding flow velocity is

$$
v_{r^{\prime}}=\frac{1}{a} \frac{\partial \psi}{\partial \phi}=v_{x c} \cos \phi, v_{\phi}=-\frac{\partial \psi}{\partial r^{\prime}}=-v_{x c} \sin \phi
$$

Integrating (5) with respect to $\phi$, we can calculate the stream function along the cylinder face as

$$
\psi=a\left(v_{x c} \sin \phi\right)
$$

The vorticity on the cylinder face can then be evaluated by solving the stream function equation, Eq. (1). The ideal fluid solution is used as the boundary condition of stream function at the outer boundary, i.e.

$$
\psi=v_{o}\left(a_{r}-\frac{a^{2}}{a_{r}}\right) \sin \theta \& \omega=0
$$

At $T=0+$, the stream function on the cylinder surface is known, i.e. Eq. (7), the rest part of the stream function is obtained by solving (1). On the contrary, the vorticity of the whole flow field is zero except that on the cylinder face which is evaluated by solving (1) with no-slip boundary condition on cylinder face. Also shown in the definition sketch, $b_{2}$ is the distance between outer boundary and the instant center of the moving circular cylinder and can be shown and expressed as

$$
b_{2}(\phi, t)=-\delta_{x}(t) \cos \phi+\left\{a_{r}^{2}-\left[\delta_{x}(t) \sin \phi\right]^{2}\right\}^{\frac{1}{2}}
$$

The first of the following equations is used to remove the 

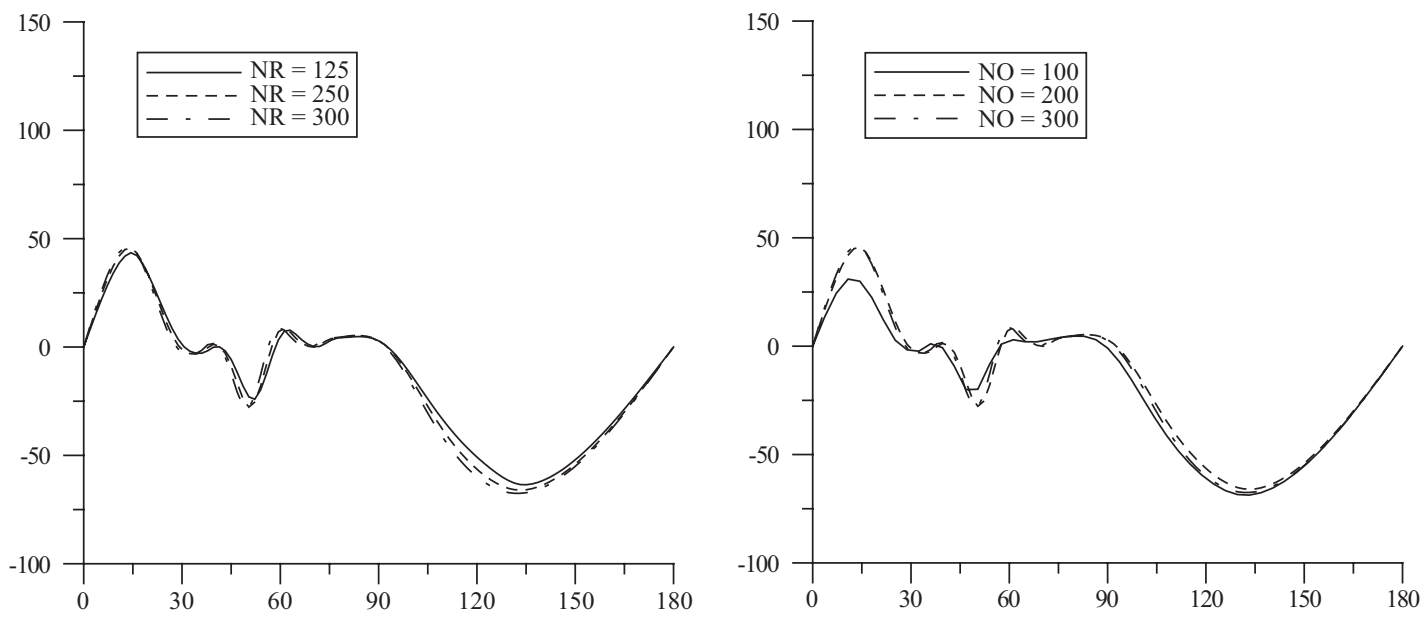

Fig. 2. The surface vorticity obtained by using various mesh-size arrangement.

dependence of $b_{2}$ on time (Hung [9] and Chen [4]).

$$
r^{*}=\frac{r^{\prime}-a}{b_{2}(\phi, t)-a} \quad \Phi=\frac{\phi}{\pi}
$$

By the first transformation, the cylinder face is mapped onto $r^{*}=0$ and the outer boundary onto $r^{*}=1$. And the second transformation would map $\phi$ onto a region $\Phi=0$ and 2. Thus, the time-dependent moving cylinder face is transformed to a fixed value and the whole computational domain is mapped onto a rectangular region. The coordinates $r^{*}$ is further transformed to produce finer grid sizes near the cylinder face. That is

$$
R=\beta_{1}+\left(r^{*}-\beta_{1}\right) e^{k_{1} r^{*}\left(r^{*}-1\right)}
$$

where $\beta_{1}$ and $k_{1}$ are the coefficients used to control grid sizes.

\section{NUMERICAL METHOD}

The solution procedures can be briefly described below. Solve the stream function equation, and then evaluate the vorticity by substituting the stream function into vorticity transport equation. Since two equations are coupled, iteration is needed to achieve acceptable convergence condition. In the two-dimensional analysis, the fluid flow is solved in a rectangular mesh network in the transformed domain (R- $\Phi)$. The finite difference method is used in the analysis and central difference is used in both space and time derivatives. And the staggered grid C-type system is used in the present analysis. As mentioned earlier, all the time derivative terms are using central time-difference approximation. However, for each time step, the explicit method is used at the first iteration to obtain a pre-condition. The Crank-Nicolson method is, thereafter, used to solve the time dependent terms at each time step. Equations (1) and (2) will be written in finite difference form and rearranged as systems of algebraic equations. Equation (2) contains nonlinear terms, after the stream function is obtained from (1), we may regard (2) as a linear equation and the Gauss-Seidel method is used to solve the systems of linear equations. The convergence conditions shown as follow are used to assure acceptable accuracy, i.e. $\left|\Psi_{m+1}-\Psi_{m}\right| \leq 10^{-6}$ and $\left|\omega_{m+1}-\omega_{m}\right| \leq 10^{-10}$ where the subscripts represent the iteration numbers. The detailed numerical algorithm is given below and also can be found in Chen [4] and Chen and Nokes [5].

1. Specify the initial conditions.

2. Stretch the grid size and calculate the coefficients of finite difference equations.

3. Solve the Poisson equation of stream-function at $T=$ $n \Delta t$.

4. Evaluate the vorticity by substituting the stream function into vorticity transport equation.

5. Solve the Poisson equation of stream-function at $T=(n+$ $1 / 2) \Delta t$.

6. Repeat steps 4 and 5 while the convergence condition is not satisfied yet.

7. Calculate the velocity for flow fluid.

8. Calculate the forces of cylinder.

9. Back to step 2 for next time step.

\section{RESULTS AND DISCUSSIONS}

A simulation of flow past a fixed circular cylinder for $R e=$ 3,000 is used to check the sensitivities of mesh size and time increment. Large computational domain is given as $r_{\infty}=25$ a. To make the problem computationally more tractable, the origin of the center of the cylinder is shift to $\mathrm{x}=-10$ a and the outer boundary at the downstream is actually 35 a away from the cylinder so that the cylinder has only a tiny effect on the flow. In order to prevent numerical instabilities, the CourantFriedrichs-Lewy condition and the necessary condition for 


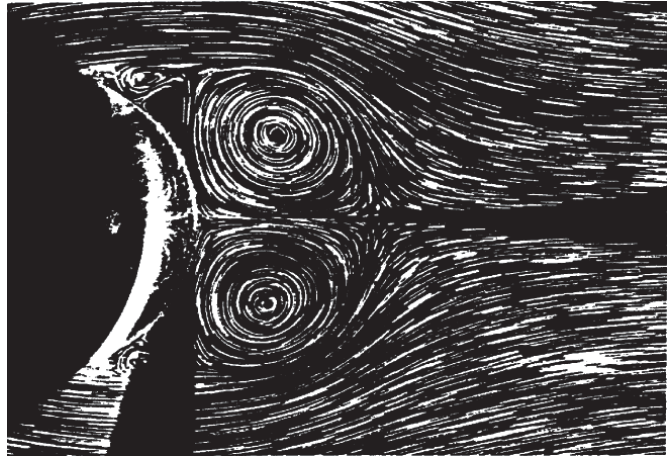

(a)

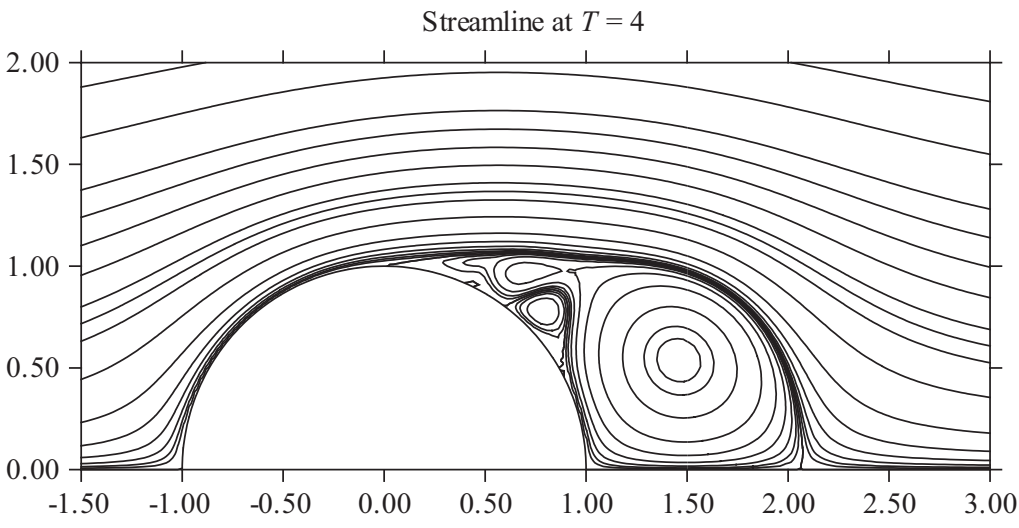

(b)

Fig. 3. Comparison of streamline patterns for $R e=3,000$, at $T=4$; (a) Ta Phuoc Loc \& Bouard [14] flow visualization, (b) present analysis.

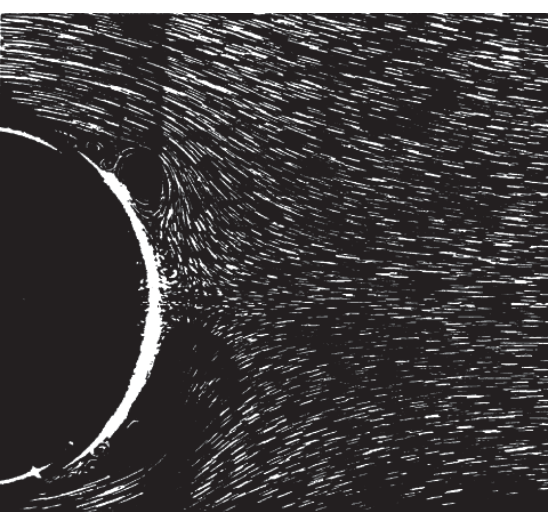

(a)

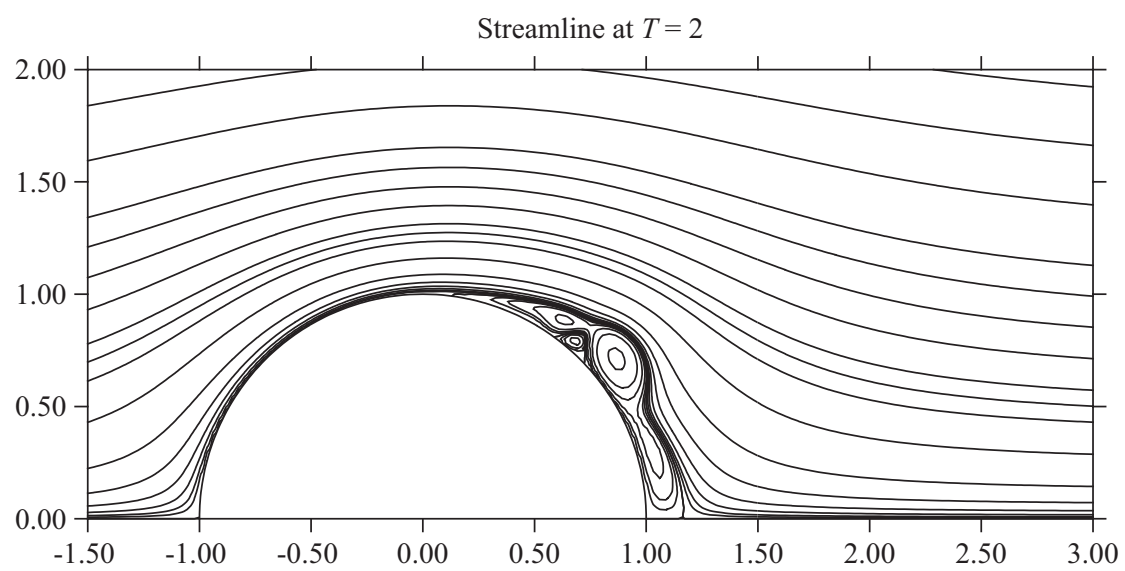

(b)

Fig. 4. Comparison of streamline patterns for $R e=9,500$, at $T=2$; (a) Ta Phuoc Loc \& Bouard [14] flow visualization, (b) present analysis.

Table 1. The parallel computation time and communication time with various numbers of CPU.

\begin{tabular}{|c|c|c|c|c|}
\hline $\begin{array}{c}\text { Numbers } \\
\text { of CPU }\end{array}$ & $\begin{array}{c}\text { Sequential } \\
\text { time } \\
(\mathrm{sec})\end{array}$ & $\begin{array}{c}\text { Parallel } \\
\text { computation } \\
\text { time } \\
(\mathrm{sec})\end{array}$ & $\begin{array}{c}\text { Parallel } \\
\text { communication } \\
\text { time } \\
(\mathrm{sec})\end{array}$ & $\begin{array}{c}\text { Total } \\
\text { time } \\
(\mathrm{sec})\end{array}$ \\
\hline 1 & 1.79 & 2273.19 & 0.00 & 2274.98 \\
\hline 2 & 1.74 & 1140.96 & 47.04 & 1189.74 \\
\hline 4 & 1.72 & 572.78 & 57.80 & 632.30 \\
\hline 8 & 1.72 & 287.87 & 62.92 & 352.51 \\
\hline
\end{tabular}

stability is also dictated by the restriction on the grid Fourier numbers. However, in order to assure the numerical accuracy, parametric study of the mesh size requirement and time increment was made. Figure 2 plots the comparison of the surface vorticity distribution along cylinder obtained by various mesh-size arrangement and for stability criteria and numerical accuracy, $\Delta R=1 / 250$ with $k_{1}=3, \Delta \Phi=1 / 200$ and $\Delta T=0.001$ are used in the present computation. The present numerical code is written in a parallel computing mode on an IBM SP2 supercomputer with eight CPU in all the simulation cases. The parallel computation time and communication time with various CPU are listed in the following Table 1.

\section{Benchmark Study}

To validate the proposed numerical method, the well-known numerical results and experimental measurements are compared with numerical results obtained by present numerical scheme. The phenomenon $\alpha$ is simulated in the present study for $R e=3,000$, while phenomenon $\beta$ is simulated for $R e=$ 9,500. For $R e=3,000$, Fig. 3 compares the present numerical results and the results reported by [14], and good agreements are noted. To generate phenomenon $\beta$, the Reynolds number is increased to 9,500, Fig. 4 lists the comparison of the present numerical results and those of PL\&B, the agreement is also very good. Further numerical validation was made according to the wake-size obtained in the present numerical model and those reported by [14] and [2] and the comparison is listed in Table 2. 
Table 2. The comparison of the wake-length and wakeheight of various reports.

\begin{tabular}{|l|c|c|}
\hline \multicolumn{1}{|c|}{ Comparison items } & $\begin{array}{c}\text { main wake length/ } \\
\text { radius }\end{array}$ & $\begin{array}{c}\text { main wake height/ } \\
\text { radius }\end{array}$ \\
\hline $\begin{array}{l}\text { Bouard and Coutanceau } \\
\text { [2] Exp. }\end{array}$ & 1.181 & 1.101 \\
\hline $\begin{array}{l}\text { Ta Phuoc Loc [14] } \\
\text { Numerical }\end{array}$ & 1.079 & 1.066 \\
\hline Present paper & 1.109 & 1.118 \\
\hline
\end{tabular}

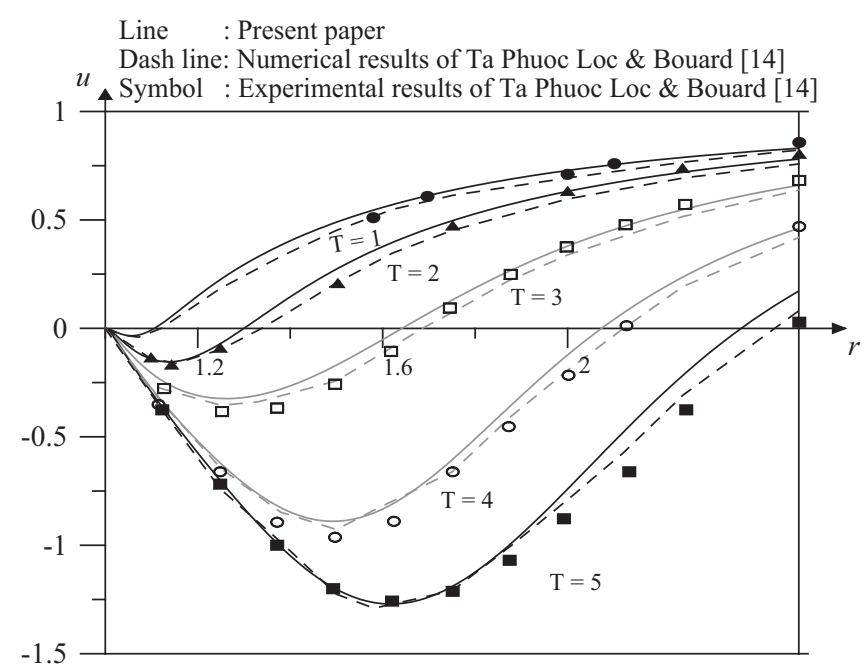

Fig. 5. The comparison of the velocity component $u$ behind the cylinder obtained by various researchers. $R e=3,000$; dashed line: $\mathrm{Nu}$ merical results of Ta Phuoc Loc \& Bouard [14], symbol line: Experimental results of Ta Phuoc Loc \& Bouard [14], solid line: present numerical results; $\bigcirc: T=1 \Delta: T=2 \square: T=3 \bigcirc: T=4$ 口: $T=5$ )

Figure 5 illustrates the comparison of the velocity component $u$ behind the cylinder obtained by various researchers. The excellent agreement can be found in the figure and the above several comparisons show the acceptance of the accuracy of the results obtained in the present numerical scheme.

\section{Flow Over a Back-Forth Oscillating Circular Cylinder}

The cross flow and cylinder are set to suddenly move at the same time. The velocity of the cross flow is a constant and is denoted as $v_{o}$, the velocity of oscillating cylinder is given as $u(t)=-\beta v_{o} \cos (\pi \varepsilon t)$, where $\varepsilon$ is frequency of oscillating cylinder and $\beta$ is a parameter to define the velocity amplitude of the moving cylinder. If the cylinder is motionless, the vortex production is basically resulted from the cross flow, when the cylinder is in motion, the vortex production will be enhanced or reduced depending on the moving direction of the cylinder. When the direction of moving direction of the cylinder is opposite to that of cross flow, the relative velocity between cylinder and the cross flow increases and the vortex production is enhanced accordingly. On the contrary, when they are in the same directions, the relative velocity between the cross flow and cylinder decreases and the vortex production is reduced. Besides, the cylinder motion will cause the splitting of the vortex around the cylinder and the periodic flow pattern will be developed as $T$ increases.

\section{1) Streamline Patterns Development ( $\beta=1, \varepsilon=1, \operatorname{Re}=3,000$ )}

For $\beta=1$, the cylinder experiences a higher Reynolds number. The equivalent $R e$, denoted as $e R e$, is equal to 6,000 at $T=0+$. There are four stages in one cycle of harmonic cylinder motion, in the first stage $(T=2 n$ to $2 n+0.5), e R e=$ 6,000 to 3,000 ; in the second stage $(T=2 n+0.5$ to $2 n+1)$, $e R e=3,000$ to $0 ;$ in the third stage $(T=2 n+1$ to $2 n+1.5)$, $e R e=0$ to 3,000 and in the fourth stage; $(T=2 n+1.5$ to $T=$ $2 n+2) e R e=3,000$ to 6,000 .

At the onset of the motions, the flow field is basically irrotational and the flow can be modeled as potential flows governed by a Laplace's equation. Thus, at the onset of the motion, the streamline patterns of the present problem can be divided into two parts: one is the flow over a fixed cylinder and the other is the cylinder moving against a still water field. According to method of superposition, when the cylinder is impulsively moving westward with a speed $v_{o}$, the corresponding stream function can be easily determined as $\psi=v_{o} r\left(1-2 a^{2} / r^{2}\right) \sin \theta$ which is equivalent to the stream function of flow pass a circular cylinder with a radius of $\sqrt{2} a$. As shown in Fig. 6 , the impulsively moving circular cylinder results in streamline pattern looks like flow across a larger cylinder whose size is $1.414 a$ (see the streamline pattern of $T=0+$ ). At $T=0.5$, the velocity of cylinder is zero and $e R e=3,000$, a clear re-circulating wake occurs at the rear part of the cylinder. As $T=1$, the cylinder moves in a same speed of the cross flow, the equivalent Reynolds number becomes zero and the streamline patterns are almost parallel except minor kink at cylinder face ranging from $\theta=0$ to $\theta=110^{\circ}$. The kink is due to the birth of the vorticity at the cylinder face. In the third stage, when $T>1$, the cylinder keeps moving eastward with decreasing speed and the cylinder presses the primary vortex. The negative vorticity along cylinder face also decreases and the kink reduces too and the separation point moves to rear cylinder face. At $T=$ 1.5 , the recirculating zone appears again but the wake is squeezed and separates into two parts: a smaller one, E1, nears the separation point (at $\phi=104^{\circ}$ ) and a larger one, E2, close to the rear stagnation point. In the fourth stage, from $T=1.5$ to 2 , the cylinder motion switches direction and $e R e$ also increases. The pseudo-cylinder appears again but its shape is not a circular and it is distorted by E1 and E2. The generation of positive and negative vortex alternatively and shedding develops in the later oscillation period.

\section{2) The Separation Points ( $\beta=1, \varepsilon=1, \operatorname{Re}=3,000)$}

The separation points are close related to the moving direction and the instantaneous location of the cylinder. Figure 7 depicts the separation point verse time. As time increases, the 


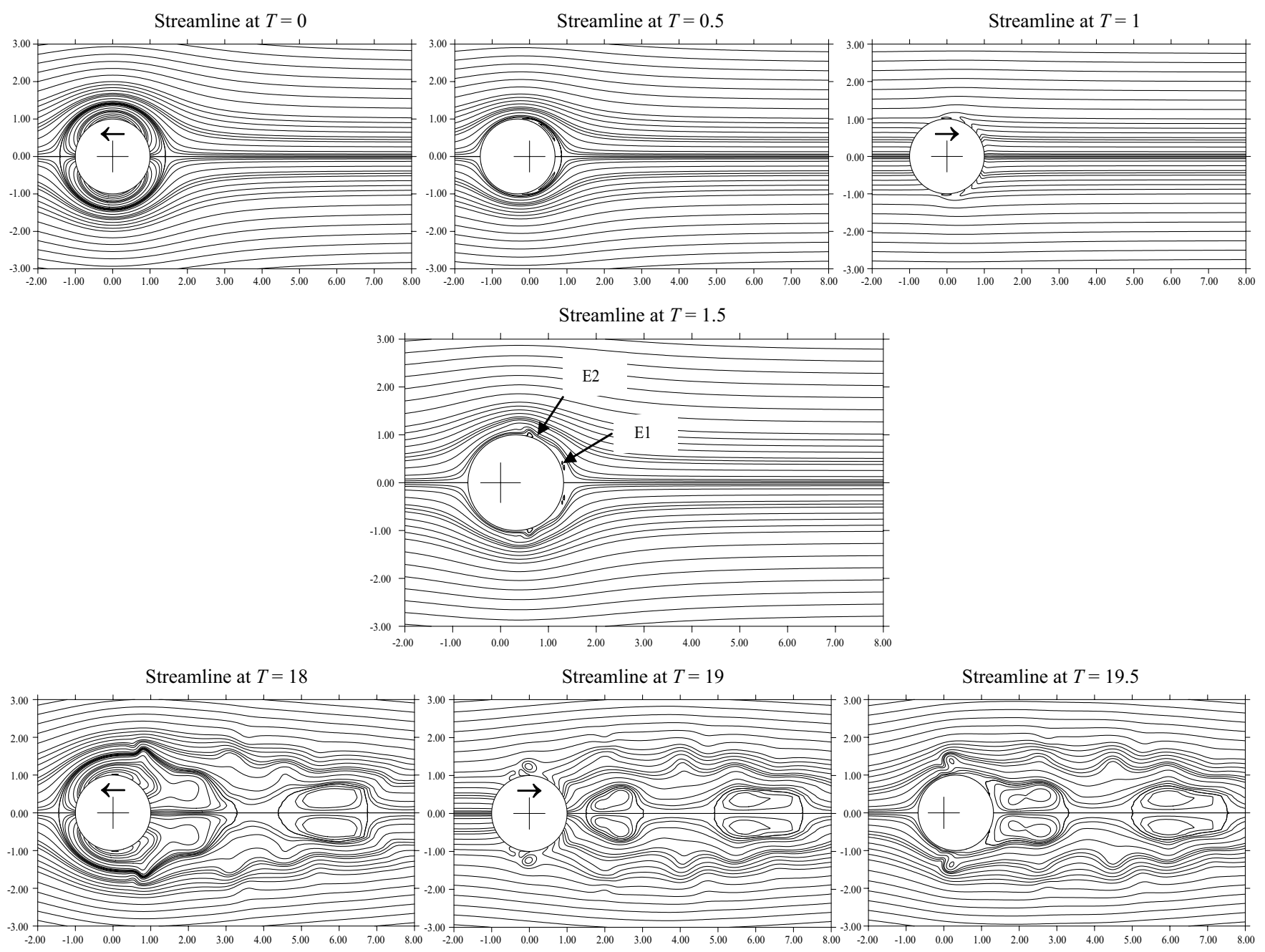

Fig. 6. The patterns of the streamlines of $R e=3,000, \beta=1$, and $\varepsilon=1$.

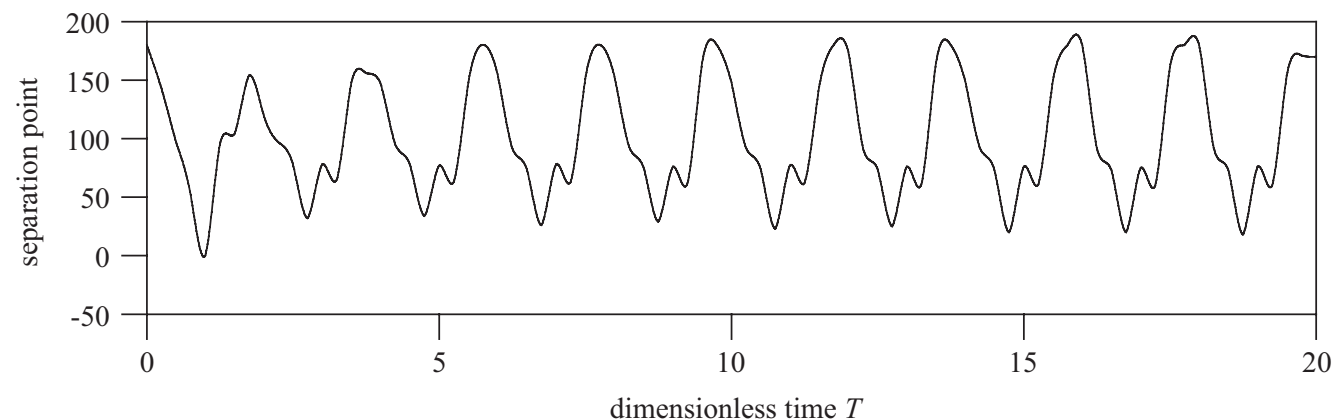

Fig. 7. Separation point position versus dimensionless time.

separation point rapidly moves more than 45 degree forward to $\theta=143^{\circ}$ when $T=0.25$. At $T=0.5$, the separation point moves to almost the midsection of the cylinder surface, to $\theta=$ $97^{\circ}$. As $T>0.5$, the cylinder moves eastward and the separation point travels further forward to $\theta=52^{\circ}$ at $T=0.75$. When cylinder moves back to origin at $T=1.0$, the cylinder has the same speed of the cross flow and the separation also moves back to the front stagnation point. However, at the later elapsed time up to $T=50$, the separation point never reaches the front stagnation point again. In the third stage, the position of cylinder is at the right of the origin and the negative vorticity shrinks to $\theta=95^{\circ}$ at $T=1.25$. In the fourth stage, the cylinder moves westward and the center of the cylinder is also at the right of the origin, and the separation point remains at 


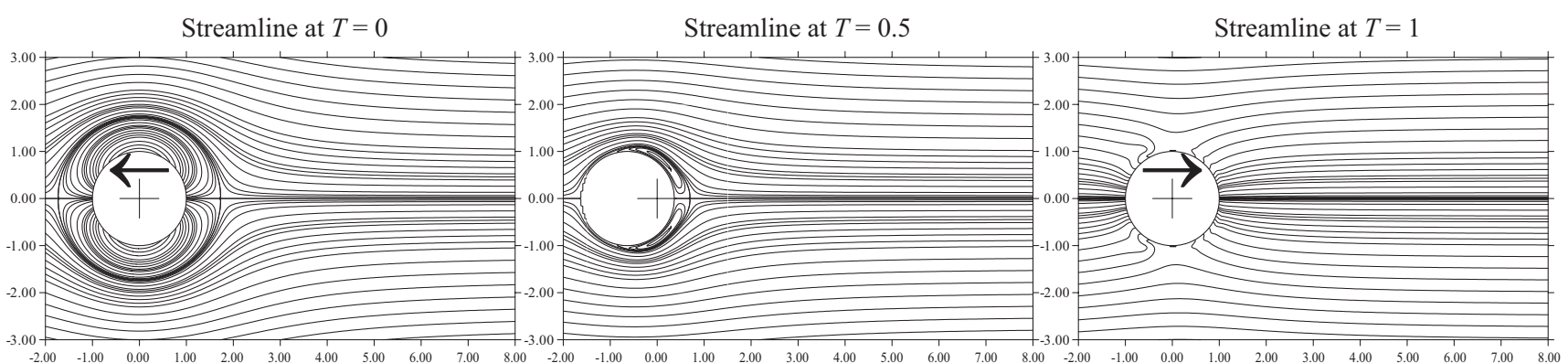

Streamline at $T=7.5$
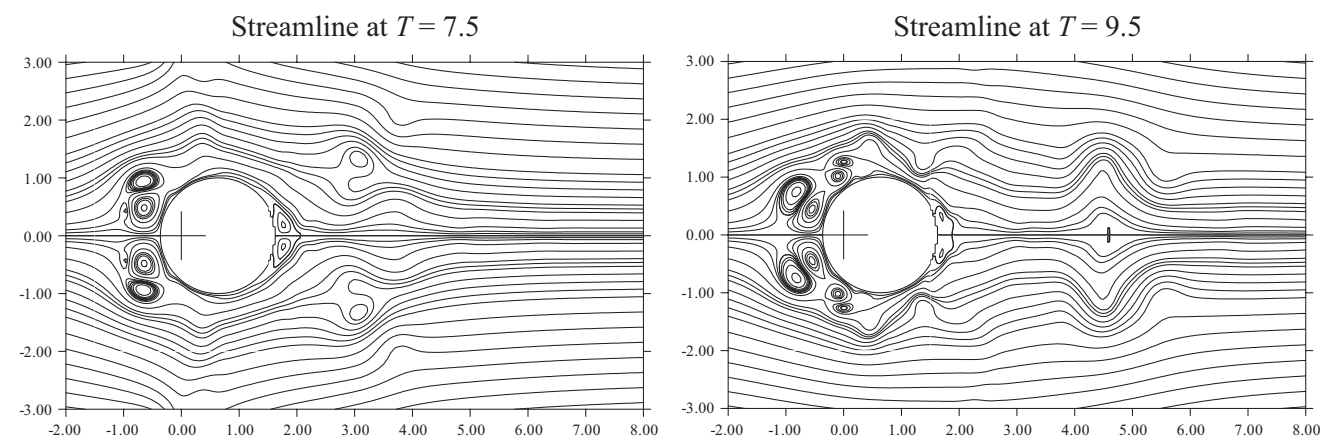

Fig. 8. The patterns of the streamlines and vorticity contour at $R e=3,000, \beta=2$, and $\varepsilon=1$.

Streamline at $T=0$
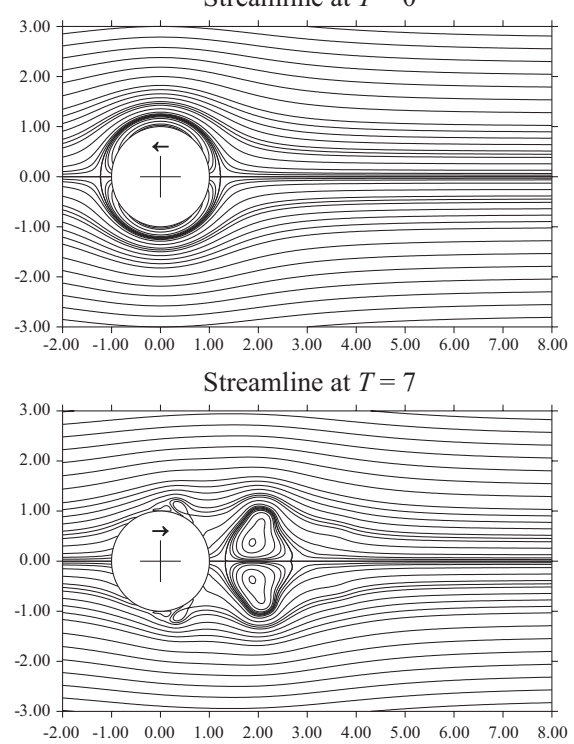

Streamline at $T=1$

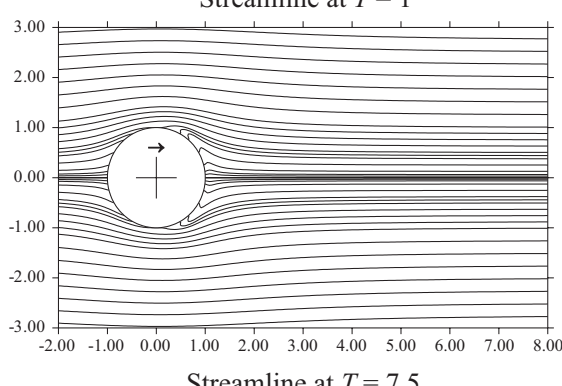

Streamline at $T=7.5$

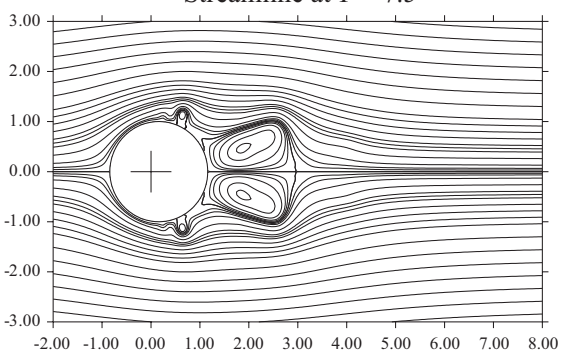

Streamline at $T=5.5$

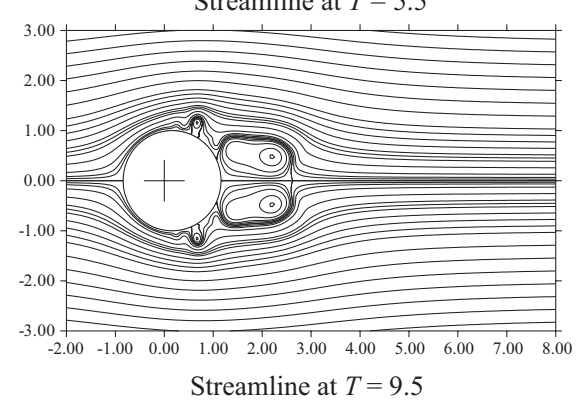

Streamline at $T=9.5$

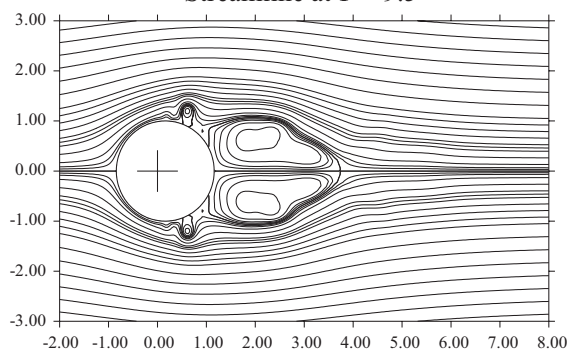

Fig. 9. The patterns of the streamlines and vorticity contour at $R e=3,000, \beta=0.5$, and $\varepsilon=1$.

rear part of the cylinder face. The phenomenon is periodically presented and harmonic behavior can be observed after $T$ is greater than 10 .

\section{3) The Results for $\varepsilon=1$ with Various $\beta$ Values}

In this section, the streamline patterns of various $\beta$ with constant $\varepsilon$ are studied. For $\beta=2$ and $T=0, e R e=9,000$, the diameter of pseudo-cylinder is $\sqrt{3} a$ (see Fig. 8). The wakes occurs at the front and also at the rear of cylinder. At $T=0.5$, the vortex at rear part of the cylinder is much stronger than that for $\beta=1$. When $T=1$, the cylinder seems experiencing a reversed cross flow and the reversed cross flow results in a pair of vortex at the front side of the cylinder.

For $\beta=0.5$, the maximum oscillating speed of cylinder is only one half of the speed of cross flow. The cross flow dominates the flow development. At $T=0, e R e=4,500$, the size of the pseudo-cylinder is smaller and is equal to $1.225 a(=\sqrt{1.5} a)$, see Fig. $9(T=0+)$. At $T=0.5$, the vortex at the rear part of cylinder is also smaller than that of $\beta=1$. At $T=1$, the speed 


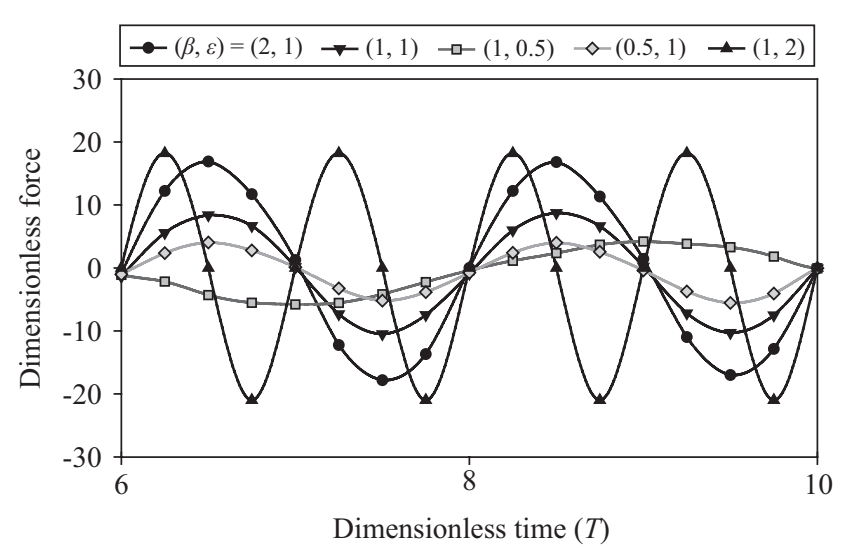

Fig. 10. Comparison of calculated dimensionless forces of various exciting frequencies and velocities of cylinder motion.

of the cylinder is $0.5 v_{o}$, the speed of equivalent cross flow is reduced but does not vanish and the streamlines around cylinder are still in curved shapes. The vortex generated at the rear of cylinder is smaller in size and strength at the earlier cycles. Besides, no vortex is generated at the front face of the cylinder. The vortex at the rear of the cylinder do not leave the cylinder up to $T=10$.

\section{4) The Forces on Cylinder}

For very low Reynolds number flows, the inertia forces on cylinder may be neglected, and the drag force on a cylinder is basically a friction drag. As the Reynolds number increases, the drag is a combination of friction and pressure drag as a result of flow separation. The relative contribution of friction drag decreases as Reynolds number increases, and the friction drag is approximately 5 percent of the total drag when $R e=$ 1,000. Figure 10 plots the dimensionless pressure drag and friction drag on cylinder versus time. The corresponding lift forces are negligible small and are not shown in the figure. The oscillating acceleration will enhance and even dominate the contribution to pressure drag, which is sum of form drag $\left(F_{d}\right)$ and inertial drag $\left(F_{i}\right)$. The relative velocity between cross flow and cylinder motion would be the major factor to determine the form drag and the friction drag on the cylinder. The well-known Morison's equation for predicting in-line forces on a circular cylinder in the cross flow with constant velocity = $v_{o}$, can be expressed as

$$
F_{x}=-F_{d}-F_{i}=-\rho a C_{d}\left(v_{o}-u\right)\left|\left(v_{o}-u\right)\right|-\rho \pi a 2 C_{m} \ddot{u}
$$

where $C_{d}$ is the drag coefficient and $C_{m}$ is the inertia coefficient, $u$ and $u$ are, respectively, the acceleration and velocity of the cylinder motion. Figure 11 shows the comparison of the calculated drag forces and that predicted by the Morison's equation and the agreement is very well.

\section{CONCLUSION}

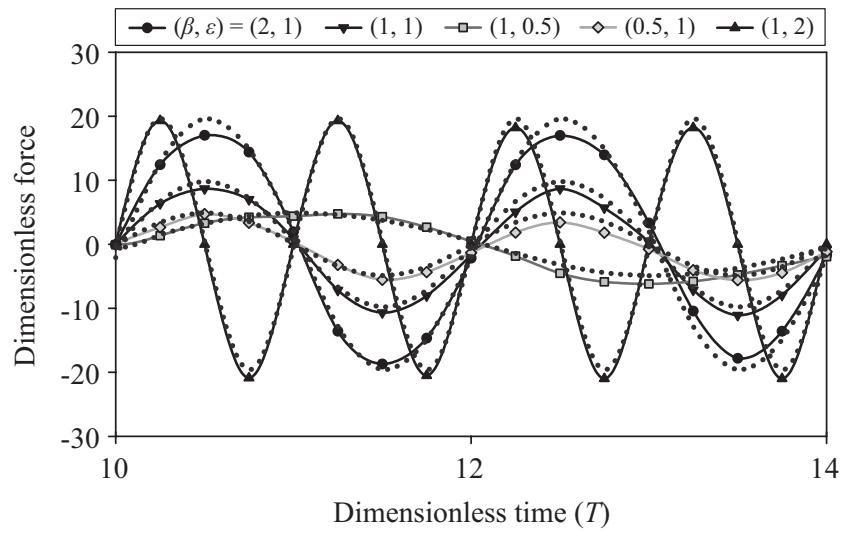

Fig. 11. Comparison of calculated dimensionless forces (solid line) versus forces predicted by Morison's equations (dotted line).

A time-independent finite difference method was used to simulate the viscous flow across a stream-wise oscillation circular cylinder. The detailed development of the streamline patterns was described. The following conclusions are reached:

1. The numerical results were validated by the reported experimental measurements and the accuracy of the results obtained in this study is good.

2. At the onset of the cylinder motion, a pseudo-cylinder was found numerically as well as theoretically.

3. The separation point is related to the location of the cylinder and the oscillation stage. The position of the separation point is basically periodic phenomenon, while minor nonperiodic cycle-to cycle variation occurs in the second and fourth stages.

4. The results of constant frequency of excitation with various exciting amplitude of cylinder velocities were simulated. For $\beta=2$, the amplitude of the cylinder velocity is two times larger than uniform velocity of cross flow, a pair of vortex occurs in the front side of the cylinder. The pairvortex is generated by the cylinder as it is moving in eastern direction since the cylinder is experienced with uniform flow passing it from east to west.

5. For the smaller cylinder velocity, the streamline development is dominated by the cross flow. The vortex development is smaller both in size and strength and the separation points are never across the north and south poles of the cylinder.

6. The developed finite different difference method can be extended to study the viscous flow passing an arbitrary moving circular cylinder.

\section{ACKNOWLEDGMENTS}

This study is partially supported by Nation Science Council of ROC under a grant NSC-90-2611-E-110-001 and NSC-962211-E-110-107-MY3. 


\section{REFERENCES}

1. Blasius, H., "Grenzschichten in flüssikeiten mit kleiner reibug," Zeitschrift für Angewandte Mathematik und Physik, Vol. 56, pp. 1-37 [English translation in NACA Technical Memo. 1256] (1908).

2. Bouard, R. and Coutanceau, M. "The early stage of development of the wake behind an impulsively started cylinder for $40<\mathrm{Re}<10^{4}$," Journal of Fluid Mechanics, Vol. 101, pp. 583-607 (1980).

3. Chang, C. C. and Chern, R. L., "A numerical study of flow around an impulsively started circular cylinder by a deterministic vortex method," Journal of Fluid Mechanics, Vol. 233, pp. 243-263 (1991).

4. Chen, B. F., "3D nonlinear hydrodynamic analysis of vertical cylinder during earthquakes, I: Rigid motion,” Journal of Engineering Mechanics, Vol. 123, No. 5, pp. 458-465 (1997).

5. Chen, B. F. and Nokes, R., "Time-independent finite difference analysis of fully non-linear and viscous fluid sloshing in a rectangular tank," Journal of Computational Physics, Vol. 209, pp. 47-81 (2005).

6. Collins, W. M. and Dennis, S. C. R., "The initial flow past an impulsively started circular cylinder," The Quarterly Journal of Mechanics and Applied Mathematics, Vol. 26, pp. 53-75 (1973).

7. Collins, W. M. and Dennis, S. C. R., "Flow past an impulsively started circular cylinder," Journal of Fluid Mechanics, Vol. 60, pp. 105-127 (1973).

8. Dütsch, H., Durst, F., Becker, S., and Lienhart, H., "Low-Reynoldsnumber flow around an oscillating circular cylinder at low KeuleganCarpenter numbers," Journal of Fluid Mechanics, Vol. 360, pp. 249-271 (1998).

9. Hung, T. K., "Forcing function in Navier-Stokes equations," Journal of Engineering Mechanics Division, Vol. 107, No. 3, pp. 643-648 (1981).

10. Justesen, P., "A numerical study of oscillating flow around a circular cylinder," Journal of Fluid Mechanics, Vol. 222, pp. 157-196 (1991).

11. Payne, R. B., "Calculations of unsteady viscous flow past a circular cylinder," Journal of Fluid Mechanics, Vol. 4, pp. 81-86 (1958).

12. Prandtl, L., "The magnus effect and windpowered ships," Naturwissenschaften, Vol. 13, pp. 93-108 (1925).

13. Ta Phuoc Loc "Numerical analysis of unsteady secondary vortices generated by an impulsively started circular," Journal of Fluid Mechanics, Vol. 100, pp. 111-128 (1980).

14. Ta Phuoc Loc and Bouard, R., "Numerical solution of the early stage of the unsteady viscous flow around a circular cylinder: A comparison with experimental visualization and measurement," Journal of Fluid Mechanics, Vol. 160, pp. 93-117 (1985). 